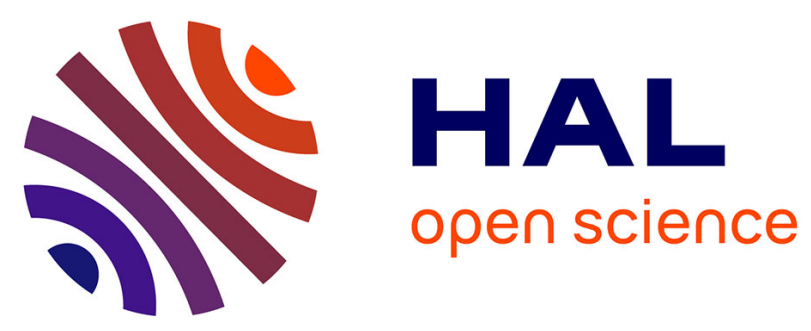

\title{
Surfactant Protection Efficacy at Surfaces Varies with the Nature of Hydrophobic Materials
}

Guillaume Lefebvre, Antoine Maze, Rosa Alvarez-Palencia Jimenez, Franz Bruckert, Vasco Filipe, Sylvain Huille, Marianne Weidenhaupt

\section{- To cite this version:}

Guillaume Lefebvre, Antoine Maze, Rosa Alvarez-Palencia Jimenez, Franz Bruckert, Vasco Filipe, et al.. Surfactant Protection Efficacy at Surfaces Varies with the Nature of Hydrophobic Materials. Pharmaceutical Research, 2021, 38 (12), pp.2157 - 2166. 10.1007/s11095-021-03133-6 . hal-03522379

\author{
HAL Id: hal-03522379 \\ https://hal.science/hal-03522379
}

Submitted on 25 Jan 2022

HAL is a multi-disciplinary open access archive for the deposit and dissemination of scientific research documents, whether they are published or not. The documents may come from teaching and research institutions in France or abroad, or from public or private research centers.
L'archive ouverte pluridisciplinaire HAL, est destinée au dépôt et à la diffusion de documents scientifiques de niveau recherche, publiés ou non, émanant des établissements d'enseignement et de recherche français ou étrangers, des laboratoires publics ou privés. 


\section{Surfactant protection efficacy at surfaces varies with the nature of \\ hydrophobic materials}

Guillaume Lefebvre $^{\mathrm{a}, \mathrm{b}}$, Antoine Maze ${ }^{\mathrm{a}}$, Rosa Alvarez- Palencia Jimenez ${ }^{\mathrm{a}, \mathrm{b}}$, Franz Bruckert ${ }^{\mathrm{a}}$, Vasco Filipe $^{\mathrm{b}}$, Sylvain Huille ${ }^{\mathrm{b}}$ and Marianne Weidenhaupt ${ }^{\mathrm{a}^{\dagger}}$

a, Univ. Grenoble Alpes, CNRS, Grenoble INP*, LMGP, 38000 Grenoble, France

*Institute of Engineering Univ. Grenoble Alpes

b, Sanofi, 94400 Vitry-sur-Seine, France

Keywords : monoclonal antibody, surface adsorption, formulation optimization, surfactant protection, plastic materials

${ }^{\dagger}$ corresponding author:

Marianne Weidenhaupt

Phelma Minatec LMGP

3 parvis Louis Neel

CS 50257

F- 38016 GRENOBLE cedex 1

France

tel : ++ 33456529335

email : marianne.weidenhaupt@grenoble-inp.fr 


\section{Abstract}

\section{Objective}

Monoclonal antibodies are in contact with many different materials throughout their life cycle from production to patient administration. Plastic surfaces are commonly found in single use bags, syringes, perfusion bags and tubing and their hydrophobic nature makes them particularly prone for adsorption of therapeutic proteins. The addition of surfactants in therapeutic formulations aims at minimizing surface and interface adsorption of the active molecules. However, their protection efficacy related to the nature of the plastic material is still poorly investigated.

\section{Methods}

We use real-time surface-sensitive techniques and immunosorbent assays, to quantify surfactant and monoclonal antibody adsorption on hydrophobic model surfaces and different plastic polymers to analyse the effect of material surface properties on the level of surfactant protection.

\section{Results}

We show that Polysorbate 80 protects monoclonal antibodies significantly better from adsorption on a polystyrene surface than on a hexadecane self-assembled monolayer, used as a model surface with similar hydrophobicity. This enhanced protective effect on polystyrene is observed for different antibodies and also other surfactants, and its extent depends on the surfactant concentration for a given antibody concentration. A comparative adsorption study allows ranking different in-use plastics and highlights the dependence of Polysorbate 80 protection efficacy on the nature of the plastic material.

\section{Conclusion}


This study demonstrates that, beyond hydrophobicity, the nature of plastic polymer surfaces affects surfactant adsorption and thereby impacts their protection efficacy in therapeutic antibody formulations.

229 words 


\section{Introduction}

Monoclonal antibodies (mAbs) are complex proteins that have become increasingly important due to their numerous therapeutic applications, in autoimmune diseases or cancers [1]. The recent development of new mAb formats, such as bispecific antibodies and conjugated antibodies, further increases the interest of medical research for this kind of drug [2].

During production, transport in primary packaging, like vials or prefilled syringes, or administration with intravenous (IV) infusion bags, monoclonal antibodies may adsorb at the liquid-solid interface and form aggregates in solution that can bring safety concerns [3]. However, while aggregate formation can be mitigated and easily monitored during mAb production, it is more difficult to predict the behaviour of therapeutic proteins during the transport, dilution and administration stages. In order to avoid any mAb surface adsorption during these steps, it is common to add surfactants to the drug formulation. Surfactants have the ability to protect therapeutic proteins by competitive adsorption at interfaces [4]. Polysorbate 80 (PS80), Polysorbate 20 (PS20) and Poloxamer 188 (P188) to a lower extent, are widely used in almost $90 \%$ of all marketed mAb formulations [5].

It has been shown that mAbs can adsorb to materials such as plastics, which are used for the manufacture of vials, syringe barrels, IV bags or tubing $[6,7]$. The sensing techniques used in interface adsorption studies are often not directly compatible with the plastic materials in contact with therapeutic solutions. Instead these studies are conducted on wellcontrolled hydrophobic surface functionalizations, mimicking the overall hydrophobic character of plastics $[8,9]$.

However, plastic materials are not defined exclusively by their hydrophobicity. They are obtained by more or less ordered polymerisation of repeated monomer units using 
processes involving heat, pressure and the addition of catalysts and plasticizer. Their nature and fabrication processes allow tuning different functionalities, such as flexibility, moisture permeation, transparency, collapsibility and compatibility with heat sterilisation, which are relevant in medical devices [10]. The most used ones in medical applications are polystyrene, polyolefins (polypropylene, polyethylene), polyvinylchloride and polycarbonate [11].

The influence of the properties of different plastics on the adsorption potential of mAbs at their surface is hitherto poorly understood. We therefore compared the adsorption and desorption kinetics of three monoclonal antibodies in the presence of PS80, PS20 and P188 on hydrophobic model surfaces (hexa and octadecane-based) and polystyrene, using surface-sensitive techniques, such as surface plasmon resonance imaging (SPRi) and quartz crystal microbalance with dissipation monitoring (QCM-d). We show that the protective effect of surfactants differs significantly on the model surfaces and on polystyrene, despite their similar hydrophobicity. Additionally, we used an enzyme-linked immunosorbent assay to study the protective effect of surfactants on a number of different plastics and show that it varies significantly with the nature of the plastic surface and depends on the mAbsurfactant ratio.

\section{Experimental}

\section{Proteins and Chemicals}

$\underline{\text { Monoclonal antibodies } \mathrm{A}, \mathrm{B} \text { and } \mathrm{C}}$

Monoclonal antibody A (mAbA) is a full IgG of subclass 1, provided by Sanofi (Paris, France) at $30 \mathrm{~g} . \mathrm{L}^{-1}$ in a formulation at $\mathrm{pH} 6.0$ containing histidine and sucrose; $\mathrm{mAbA}$ is positively charged at $\mathrm{pH} 6.0$. 
$m A b B$ is a bispecific antibody fragment construct derived from a subclass 1 lgG provided by Sanofi (Paris, France) at $0.7 \mathrm{~g} . \mathrm{L}^{-1}$ in a formulation at $\mathrm{pH} 6.0$ containing histidine, glycine and sucrose; mAbB is positively charged at $\mathrm{pH} 6.0$.

$\mathrm{mAbC}$ is a full IgG of subclass 4 , provided by Sanofi (Paris, France) at $3 \mathrm{~g} \cdot \mathrm{L}^{-1}$ in a formulation at $\mathrm{pH} 6.1$ containing histidine, sucrose and proline; $\mathrm{mAbC}$ is also positively charged at $\mathrm{pH} 6.1$. The charge and hydrophobicity characteristics of the three mAbs are published [12].

The buffers of the respective mAbs are hereafter named Bf $1 x$. Concentrated mAb aliquots were stored at $-20^{\circ} \mathrm{C}$ and thawed shortly before use. When thawed, mAb solutions were kept at $4^{\circ} \mathrm{C}$. They were diluted in their respective $\mathrm{Bf} 1 \mathrm{x}$ to the working concentrations. The addition of different surfactants to the formulations was made by spiking a concentrated solution of the surfactant to reach the desired final concentration. The concentrations of $\mathrm{mAb}$ aliquots were verified by absorbance at $280 \mathrm{~nm}$ using a Nanodrop spectrophotometer.

\section{$\underline{\text { Protein G/A- HRP conjugates }}$}

Protein G-HRP (Merck Ref 18-161) and protein A-HRP (GE Healthcare Ref NA9120V) conjugates were rehydrated at $1 \mathrm{~g} \cdot \mathrm{L}^{-1}$ in MilliQ water. Concentrated protein aliquots were stored at $-20^{\circ} \mathrm{C}$ and thawed shortly before use. When thawed, protein $\mathrm{G}$ and $\mathrm{A}$ conjugates were diluted at $250 \mu \mathrm{g} . \mathrm{L}^{-1}$ in the mAb-specific buffers.

\section{$\underline{\text { Surfactants }}$}

Polysorbate 80 (PS80) (Seppic Montanox 80ppi, MW = 1310 g.mol ${ }^{-1}$ ) and Polysorbate 20 (PS20) (Seppic Montanox 20ppi, MW = 1227 g.mol ${ }^{-1}$ ) are compliant with EP, USP and JP monographs. Polysorbates and Poloxamer 188 (P188) (Merck) were diluted at the indicated concentrations in the respective buffers (Bf1x), described above.

\section{Dynamic light scattering}


The absence of protein aggregates was verified for mAb solutions by dynamic light scattering (DLS) on a Malvern Zetasizer Nano instrument. mAbA was measured at 30 g. $\mathrm{L}^{-1}, \mathrm{mAbB}$ at 0.7 g. $\mathrm{L}^{-1}$ and $\mathrm{mAbC}$ at $3 \mathrm{~g} \cdot \mathrm{L}^{-1}$. The data were acquired using the ZSExplorer size protocol (30 acquisitions, total time 50.4s) and analysed with ZSExplorer software (general purpose model, adaptive correlation mode). From the size distribution by intensity, the hydrodynamic diameter was deduced based on the Stokes-Einstein equation and was $10 \mathrm{~nm}$ as expected for $\mathrm{mAbA}, \mathrm{mAbB}$ and $\mathrm{mAbC}$ with low polydispersity index values $(<0.13)$.

\section{Surface functionalizations}

The sensor surface of gold-coated prisms (Horiba SPRi Biochips) was functionalized according to a protocol described by Nault [13] to obtain a hydrophobic surface, coated with hexadecane thiol ( $\mathrm{C} 16-\mathrm{SH}$, Sigma-Aldrich), diluted to $1.6 \%$ in toluene and with a thiolterminated polystyrene (P10826A-SSH, Polymer source), diluted to $1 \%$ in toluene.

In the same way, gold-coated QCM-d sensors (QSX301) from Biolin Scientific (Västra Frölunda, Sweden) were functionalized to obtain a hydrophobic surface, coated with hexadecane thiol (C16-SH, Sigma-Aldrich), diluted to $1.6 \%$ in toluene. The polystyrene QCMd sensors (QSX305) were purchased from Biolin Scientific.

Borosilicate glass beads ( $1 \mathrm{~mm}$ - Z273619, Sigma-Aldrich) were cleaned by rinsing with $14.5 \mathrm{M}$ $\mathrm{NaOH}$ followed by repeated rinsing with deionized water and drying at $100^{\circ} \mathrm{C}$ for $1 \mathrm{~h}$. Cleaned glass beads were functionalized by immersion during 2 hours with gentle agitation in octadecyl methyl dichlorosilane (C18) (AB111265, $A B C R$ ), diluted to $0.1 \%$ in toluene. Then, the functionalized beads were washed with toluene, ethanol and water and cured at $100^{\circ} \mathrm{C}$ for 1 hour.

\section{Surface characterisation}


X-Ray Photoelectron spectra were recorded on a ThermoScientific K-Alpha spectrometer using a monochromatized Al $\mathrm{K} \alpha$ radiation source $(1486.6 \mathrm{eV})$, in ultra high vacuum $\left(10^{-8}\right.$ mbar) at room temperature. The X-Ray beam area (spot size) was adjusted to $400 \mu \mathrm{m}$ in diameter. Analyses were conducted at $90^{\circ}$ angles between the sample surface and the analyzer. The photoelectrons emitted were inspected with a $180^{\circ}$ double focusing hemispherical analyzer and a 128-channel detector. The spectra were acquired in the constant analyzer energy mode using a pass energy of $30 \mathrm{eV}$ and a step size of $0.1 \mathrm{eV}$ for core levels, and a $100 \mathrm{eV}$ pass energy has been used for the general survey spectrum (step $0.5 \mathrm{eV})$. The spectra shown in the figures have been averaged over 3 scans for the survey and 10 scans for the core levels. The flood gun was used to neutralize charge effects on the surface. All the binding energies were calibrated relatively to the $\mathrm{C}-\mathrm{C}$ bond component of the C 1 s core electrons at $284.6 \mathrm{eV}$ from adventitious carbon adsorbed on the sample surface. The inelastically scattered electron-related background signal has been substracted by the Shirley method. Peak analysis and decompositions were performed using Thermo Scientific Avantage software.

\section{Plastic multiwell plates}

Polystyrene (655101) and polypropylene (650201) 96-well microplates were purchased from Greiner and polycarbonate (6520), polyvinylchloride (2595), cyclic olefin copolymer (4680) and PEG-coated (3651) 96-well microplates were purchased from Corning. The water contact angles (WCA) on these plastics were measured with a drop shape analyzer (DSA100 Krüss Scientific) and were as follows: Polystyrene WCA $=88.9 \pm 3.2$; polypropylene $\mathrm{WCA}=90.0 \pm$ 2.0; polycarbonate $\mathrm{WCA}=82.2 \pm 4.9$; polyvinylchloride $\mathrm{WCA}=95.9 \pm 3.4$; cyclic olefin copolymer WCA $=85.9 \pm 1.0$. 


\section{Surface plasmon resonance imaging (SPRi)}

\section{Sensogram acquisition}

Reflectivity data were obtained with an SPRi instrument (Horiba Scientific SPRi-Lab+ ${ }^{\mathrm{TM}}$ ) using an established protocol [12]. Buffer, protein and surfactant solutions were prepared at room temperature and degassed under vacuum before use.

The amount of stably surface-adsorbed molecules was determined after rinsing with $B f 1 x$ and stabilization of the signal by measuring the resonance signal relative to the baseline and is indicated as $\triangle \mathrm{R}$. SPRi signals, recorded every 3 seconds from each spot, were used to calculate a mean value and standard deviation for each measurement. For each experimental condition, three independent experiments were performed.

\section{Quartz crystal balance with dissipation monitoring (QCM-d)}

Protein and surfactant adsorption were measured on a Q-Sense E4 system QCM-d instrument (Biolin Scientific, Västra Frölunda, Sweden). Buffer, protein and surfactant solutions were prepared at room temperature and degassed under vacuum before they were injected in the QCM-d chambers using a peristaltic pump.

Before each experiment, the crystal surface was cleaned successively by sonication in $2 \%$ SDS and absolute ethanol for 10 minutes each. The baseline was set by the frequency obtained with Bf1x, corresponding to each protein. After baseline stabilization, protein and surfactant solutions were injected at the indicated concentrations in their respective $\mathrm{Bf} 1 \mathrm{x}$. The flow rate $\left(30 \mu \mathrm{L} \cdot \mathrm{min}^{-1}\right)$ was kept constant during the experiment. Protein and surfactant solutions were injected until the frequency signal reached a steady state. The amount of stably surface-adsorbed molecules was determined after rinsing with $\mathrm{Bf} 1 \mathrm{x}$ and stabilization of the signal by measuring the frequency signal relative to the baseline and is indicated as $\Delta \mathrm{F}$. For each experimental condition, two independent experiments were performed. 


\section{Enzyme Linked Immunosorbent Assay (ELISA)}

The concept of the ELISA-based protocol relies on quantifying relative amounts of mAb that adsorb directly on different material surfaces. An enzymatically driven, colorimetric readout is obtained thanks to the specific binding of PtG/PtA-HRP. Absorbance is therefore directly proportional to the amount of surface-adsorbed mAb. We analyze absorbance values obtained for mAb surface adsorption without and with increasing amounts of surfactant. ELISA experiments were performed directly in plastic multiwell plates (PS, PP, PVC, COC and PC) and on C18-functionalized beads in PEG-coated multiwell plates, which are protein repellent. The low level of protein binding in PEG-coated multiwell plates is documented in Supplementary Figure 3a. In each PEG-well, 31 C18-coated glass beads were added. The surface area of 31 beads (diameter $=1 \mathrm{~mm}$ ) is approximatively the same as the one in contact with the solution in polystyrene or polypropylene wells when filled with $100 \mu \mathrm{L}$ solution. mAb adsorption to the hydrophobic surfaces was quantified by protein G-HRP or protein A-HRP binding using an enzymatic read-out with tetramethylbenzidine (TMB) and $\mathrm{H}_{2} \mathrm{O}_{2}$. ELISA results obtained with both $\mathrm{HRP}$ constructs gave very similar results (Supplementary Figure 3b). The results represent mean values and their respective standard deviations obtained from a minimum of 8 independent replicate wells.

$\mathrm{mAbA}, \mathrm{B}$ and $\mathrm{C}$, concentrated at $30 \mathrm{~g} \cdot \mathrm{L}^{-1}, 0.7 \mathrm{~g} \cdot \mathrm{L}^{-1}$ and $3 \mathrm{~g} \cdot \mathrm{L}^{-1}$, respectively, were mixed with different concentrations of PS80 $\left(0,0.05,0.1\right.$ and $\left.0.2 \mathrm{~g} . \mathrm{L}^{-1}\right)$ before filling the wells. The plates were filled with $100 \mu \mathrm{L}$ protein-surfactant solution per well and shaken at $900 \mathrm{rpm}$ (Heidolph Titramax vibrating platform microplate shaker) for 15 minutes. Then each well was emptied and washed 4 times with $200 \mu \mathrm{L}$ PS80 at $0.2 \mathrm{~g} \cdot \mathrm{L}^{-1}$. The wells were then filled with $100 \mu \mathrm{L}$ protein G- or protein A-HRP at $250 \mu \mathrm{g} . \mathrm{L}^{-1}$ containing $0.2 \mathrm{~g} \cdot \mathrm{L}^{-1} \mathrm{PS} 80$ and shaken at $900 \mathrm{rpm}$ for 20 minutes. Four new washing steps were performed as previously described. A tablet (1mg) 
of tetramethylbenzidine (TMB) (Sigma Aldrich Ref T5525) was diluted in $1 \mathrm{~mL}$ dimethylsulfoxide and supplemented with $9 \mathrm{~mL}$ of phosphate citrate buffer (Sigma Aldrich Ref P4809) containing $2 \mu \mathrm{L} \mathrm{H}_{2} \mathrm{O}_{2} 30 \%$. The wells were then filled with $100 \mu \mathrm{L}$ TMB $-\mathrm{H}_{2} \mathrm{O}_{2}$ substrate solution, and the reaction was stopped after 90 seconds by adding $20 \mu \mathrm{L} \mathrm{H}_{2} \mathrm{SO}_{4} 2 \mathrm{M}$ under agitation. mAb adsorption was monitored by absorbance $(\lambda=450 \mathrm{~nm})$ on a Tecan Infinite M1000 multimode microplate reader (TECAN USA, Boston, MA).

\section{Results}

\section{PS80 and mAbA adsorption differs on polystyrene and on hexadecane surfaces}

We compared the protective effect of PS80 against mAbA adsorption on two hydrophobic surfaces using two complementary surface-sensitive techniques, SPRi and QCM-d. A selfassembled hexadecane (C16) monolayer was chosen as a model hydrophobic surface (WCA = $103 \pm 2^{\circ}$ ) and polystyrene (PS) (WCA $=89 \pm 4^{\circ}$ ) as an example of a plastic polymer. Goldcoated prisms and QCM sensors were functionalized with C16 and PS as described in the experimental section or directly purchased.

After stabilization of the SPRi or QCM-d signals in buffer, PS80 was injected at $0.2 \mathrm{~g}^{-\mathrm{L}^{-1}}$ on both surfaces followed by an injection of buffer allowing to estimate the amount of irreversibly adsorbed surfactant to the surfaces (Figure $1 \mathrm{a}$ and $b$ ). The results show that PS80 remains adsorbed on both surfaces, with $\Delta R_{P S 80}^{C 16}=5 \pm 1.5 \mathrm{RU}$ and $\Delta F_{P S 80}^{C 16}=-7.5 \pm 0.5 \mathrm{~Hz}$ on $\mathrm{C} 16$ and $\Delta R_{P S 80}^{P S}=6.5 \pm 2.5 \mathrm{RU}$ and $\Delta F_{P S 80}^{P S}=-11.5 \pm 1.5 \mathrm{~Hz}$ on polystyrene, measured by SPRi and QCM-d, respectively (Table I). Both techniques thus confirm an irreversible and slightly higher surface adsorption of PS80 on PS than on C16.

Subsequently, mAbA was injected at $30 \mathrm{~g} \cdot \mathrm{L}^{-1}$ on both surfaces until the signal was stabilized and then desorbed using Bf1x. The reflectivity signals (Figure 1a) show that mAbA remains less adsorbed on PS than on C16 surfaces pre-incubated with PS80, with reflectivity 
differences equal to $2 \pm 2 \mathrm{RU}\left(\Delta R_{m A b A}^{P S}-\Delta R_{P S 80}^{P S}\right)$ and $11 \pm 0.5 \mathrm{RU}\left(\Delta R_{m A b A}^{C 16}-\Delta R_{P S 80}^{C 16}\right)$, respectively (Table I). Likewise, the level of mAbA adsorption measured by QCM-d on PS is much lower than on C16, when pre-adsorbed with PS80, with frequency differences equal to $-3.5 \pm 3 \mathrm{~Hz}\left(\Delta F_{m A b A}^{P S}-\Delta F_{P S 80}^{P S}\right)$ and $-18 \pm 0.5 \mathrm{~Hz}\left(\Delta F_{m A b A}^{C 16}-\Delta F_{P S 80}^{C 16}\right)$, respectively.

We conclude that, after a pre-treatment of PS and C16 with PS80, slightly more surfactant remains adsorbed on PS, and $\mathrm{mAbA}$ adsorbs significantly less on PS pre-coated with PS80 than on C16 pre-coated in the same way.

\section{Quantification of PS80 protective efficacy on polystyrene and hexadecane surfaces for the}

\section{formulated mAb}

We then developed a protocol to confirm the surface-dependent protection efficacy of PS80 by comparing the amount of surface-adsorbed mAbA in the presence $\left(0.2 \mathrm{~g}^{-\mathrm{L}^{-1}}, \mathrm{PS} 80\right.$ concentration used in the formulated mAbA) and absence of surfactant on PS and C16functionalized sensors. To do this, we used protein G (PtG), known to bind specifically to the Fc region of IgGs, to measure the amount of surface-adsorbed mAbA. In order to test whether mAb surface adsorption could alter the accessibility for PtG binding sites, we compared the readouts of PtA- and PtG-HRP constructs in an ELISA assay on polystyrene. Supplementary Figure $3 \mathrm{~b}$ shows that both HRP constructs have equal performances, indicating that mAbA surface adsorption is unlikely to affect binding site accessibility for PtG. The PtG reflectivity and frequency signals were measured first on surfaces precoated with PS80 alone at 0.2 g. $\mathrm{L}^{-1}$ to take into account non-specific $\mathrm{PtG}$ adsorption $\left(\Delta \mathrm{R}_{0}\right)$ (Figure 2a, Supplementary Figure 1 and Supplementary Table I). These signals were very low, demonstrating that PtG is a valid marker allowing to specifically quantify mAbA surface adsorption. We then measured the PtG reflectivity and frequency signals associated to the surface adsorption of mAbA $\left(30 \mathrm{~g} . \mathrm{L}^{-1}\right)$ without surfactant $\left(\Delta \mathrm{R}_{1}\right)$. We considered $\Delta \mathrm{R}_{1}$ as the 
value representing the maximal mAbA adsorption level on the surfaces (Figure $2 b$, Supplementary Figure 1 and Supplementary Table I). A comparison of the respective adsorption levels of mAbA on C16 and PS shows that reflectivity data (SPRi) indicate twice as much mAbA adsorption on PS than on C16 while the frequency data (QCMd) indicate similar adsorbed amounts on both surfaces (Supplementary Table I). This could be due to different PS densities on the spin-coated QCMd sensor and the PS-thiol functionalized SPR prism. Surface characterisation by XPS (Supplementary Figure 2) indeed confirms the density difference of the PS functionalisations on both sensors. For C16 surfaces, obtained by the same thiol functionalisation protocol on both SPRi and QCMd sensors, the surface densities are similar (Supplementary Figure 2).

To quantify the protective efficacy of the surfactant, we finally measured the PtG reflectivity and frequency signals obtained on the surfaces after adsorption of mAbA $\left(30 \mathrm{~g} \cdot \mathrm{L}^{-1}\right)$ in the presence of 0.2 g. $\mathrm{L}^{-1}$ PS80 (formulation conditions) $\left(\Delta R_{2}\right)$ (Figure $2 c$, Supplementary Figure 1 and Supplementary Table I). The amount of mAbA specifically adsorbed to the surfaces was quantified using the measured PtG signals and expressed as $\left(\Delta R_{2}-\Delta R_{0}\right) / \Delta R_{1}$. The results show that, on PS, PS80 can limit mAbA adsorption to $46 \%$ (by SPRi) and to $33 \%$ (by QCM-d), compared to the mAbA adsorption level in the absence of PS80 (Figure 2d). On the C16 surface, however, the protective efficacy of PS80 is very low and mAbA adsorption can only be reduced to $98 \%$ (by SPRi) and $91 \%$ (by QCM-d), respectively (Figure $2 \mathrm{~d}$ ). These results prove that, PS80 protects $\mathrm{mAbA}$ more effectively against adsorption on polystyrene (PS) than on a hexadecane (C16) surface.

\section{Surfactant protection of mAbs depends on the material surface}


In order to confirm and verify if the above results can be generalized to different antibodies and surfactants, we analyzed the adsorption of 3 different mAbs on the same surface chemistries using an ELISA assay. The adsorption of $\mathrm{mAbA}$ at $30 \mathrm{~g} \cdot \mathrm{L}^{-1}, \mathrm{mAbB}$ at $0.7 \mathrm{~g} \cdot \mathrm{L}^{-1}$ and $\mathrm{mAbC}$ at $3 \mathrm{~g} \cdot \mathrm{L}^{-1}$ (concentrations of formulated mAbs), was analysed as a function of PS80 concentration in a PS multi-well plate and on octadecane (C18)-functionalized glass beads in a protein-repellent polyethylene glycol (PEG)-coated multiwell plate. Note that in these experiments the surface area in contact with the solution limits the amount of protein adsorption, resulting in similar adsorption levels despite significantly different mAb bulk concentrations. On PS, the protective effect of PS80 for all mAbs tested increases with the concentration of the surfactant and is strongest at $0.2 \mathrm{~g} \cdot \mathrm{L}^{-1}$, when $\mathrm{mAbA}, \mathrm{mAbB}$ and $\mathrm{mAbC}$ remain adsorbed at $2 \%, 7.5 \%$ and $6 \%$, respectively, relative to the condition without surfactant (Figure 3a). On C18-functionalized glass beads, however, the protective effect is minimal and $\mathrm{mAbA}, \mathrm{mAbB}$ and $\mathrm{mAbC}$ remain adsorbed at $71 \%, 75 \%$ and $88 \%$, respectively, at $0.2 \mathrm{~g} \cdot \mathrm{L}^{-1}$ of surfactant (Figure 3a), relative to their adsorption level in the absence of surfactant. The amount of each mAb, adsorbing directly to PEG-coated wells, was shown to be low, relative to the amount measured on C18-functionalized beads in PEG-coated wells (17\% for mAbA, $12 \%$ for $\mathrm{mAbB}$ and $13 \%$ for mAbC, Supplementary Figure 3a). Despite different overall hydrophobicities for the three mAbs [12], these results confirm that the protective effect of PS80 is strongly dependent on the material surface.

In Figure 3b, we extend the results obtained with PS80 to PS20 and P188. On C18functionalized beads, the mAbA adsorption in the presence of $0.2 \mathrm{~g} \cdot \mathrm{L}^{-1}$ surfactant is $71 \%, 61 \%$ and 53\% for PS80, PS20 and Poloxamer 188, respectively, compared to the condition without surfactant. On PS plates, mAbA remains adsorbed at $5 \%, 3 \%$ and $9 \%$ in the presence of $0.2 \mathrm{~g} \cdot \mathrm{L}^{-1}$ of PS80, PS20 and P188, respectively, relative to the adsorption level without 
surfactant. We therefore conclude that the protective effect of surfactants differs depending on the material surface, and this is true for all antibodies and surfactants tested.

\section{The protective effect of PS80 varies on different plastic materials}

We further broadened our findings to other, commercially available, plastics, notably polypropylene (PP), polyvinylchloride (PVC), cyclic olefin copolymer (COC) and polycarbonate (PC) using the ELISA-based assay (Figure 4). The absorbance data (Figure 4a) were obtained by a colorimetric readout using PtG/PtA-HRP which specifically binds to surface-adsorbed mAbA. Absorbance levels for mAbA without surfactant are in a similar range for the different materials, except for the PC plate, on which they are significantly lower. This is due to the fact that the surface area in contact with the solution in PC plates is $50 \%$ smaller and the well volume is reduced to $50 \mu \mathrm{L}$ in these plates. In formulation conditions (30g. $\mathrm{L}^{-1} \mathrm{mAbA}$ and $0.2 \mathrm{~g} . \mathrm{L}^{-1} \mathrm{PS} 80$ ), the surfactant shows very good protection on PS and PP and PC with $1.7,4.4$ and $3.6 \% \mathrm{mAbA}$ adsorption, respectively, compared to the adsorption level without surfactant (Figure $4 a$ and b). On PVC, the amount of adsorbed mAbA is reduced to $48.4 \%$, illustrating an intermediate protection effect of PS80 (Figure 4a and b). mAbA adsorption could not be prevented by PS80 on COC plates in formulation conditions. Very similar results were also obtained with PS80 from Croda (Supplementary Figure 4), illustrating that, within pharmacopeia limits, the variations in the chemical compositions of surfactants from different suppliers do not change their protective efficacy on the tested plastics. Interestingly, the PtG-HRP construct showed a high level of non-specific binding to the COC surface in the absence of mAbA. ELISA assays on COC plates were therefore performed with a PtA-HRP construct. Both PtG- and PtA-HRP constructs showed equal performances in the ELISA assays on PS plates (Supplementary Figure 3b), indicating that mAb surface adsorption is unlikely to affect binding site accessibility for PtG. 


\section{Discussion}

The control of molecular surface interactions is pivotal in therapeutic formulation development as well as in compatibility assessment for materials in contact with blood cells in vitro and in the human body. This is particularly the case for a plethora of hydrophobic plastic materials in contact with therapeutic formulations from their production, all the way to their administration [11]. In-use plastic materials are often mimicked in experimental studies by hydrophobic model surfaces, which have the advantage to be easily implemented with interface-sensitive techniques [14], but are not fully representative of the diversity of the chemical and physical properties of plastics. Our study compares directly mAb and surfactant adsorption profiles on a self-assembled alkyl model monolayer (C16) and on polystyrene (PS) by SPRi and QCM-d. We further extend our investigations on surface adsorption and surfactant protection to commercially available plastics using an adapted

\section{ELISA protocol.}

PS80 adsorbs as expected irreversibly on the hydrophobic surfaces, slightly more on the PS surface compared to the C16 surface (Figure 1 and Table I). This corroborates the findings of Zhang and colleagues, who have demonstrated, by QCM-d and neutron reflectometry, that another polysorbate, PS20, adsorbs fast and irreversibly on a PS surface [6]. PS20 also irreversibly adsorbs on silicone oil, a hydrophobic lubricant often used in contact with therapeutic solutions [15]. Subsequent injection of $\mathrm{mAbA}$ at $30 \mathrm{~g} \cdot \mathrm{L}^{-1}$ results in the accumulation of a significantly higher mass on C16, precoated with PS80, than on PS, precoated in the same way (Figure 1 and Table I). Using PtG, which selectively binds to immunoglobulins and shows very low nonspecific binding to both C16 and PS surfaces precoated with PS80 (Figure 2), we demonstrate that, in formulation conditions, PS80 cannot prevent irreversible mAb binding on both C16 and PS, albeit in different proportions. 
The protective effect of PS80 is significantly better on PS with mAbA adsorption reduced to $46 \%$ (SPRi) and 32\% (QCM-d), relative to mAbA adsorption without surfactant (100\%). The amount of mAbA adsorbing on a C16 model surface in the same formulation conditions, is 98\% (SPRi) and $90 \%$ (QCM-d) compared to mAbA adsorption without PS80 (100\%) (Figure2). Commonly, adsorption studies compare hydrophobic and hydrophilic model surfaces when analyzing the protective effect of surfactants on protein adsorption [16]. The comparison with protective efficacy of surfactants on hydrophobic surfaces present in the literature is difficult, because individual studies present data with different molecular systems, experimental protocols and techniques. Our results differ from the conclusions of Kapp and colleagues [8], who demonstrated by QCM-d and total internal reflection fluorescence microscopy (TIRF), that pre-adsorbed PS80 on a trifluoropropyl-chloromethylsilane surface, $\left(\mathrm{WCA}=89^{\circ}\right)$, was able to prevent the adsorption of an $\operatorname{lgG} 1$ concentrated at $10 \mathrm{~g} \cdot \mathrm{L}^{-1}$. On the other hand, Couston and colleagues demonstrated by TIRF, on an octadecyl-trichlorosilane surface $\left(\mathrm{WCA}=107.1^{\circ}\right)$, that a precoating with PS80 and PS20 did not completely prevent the adsorption of an IgG1 at significantly lower concentrations of $0.01 \mathrm{~g} . \mathrm{L}^{-1}[9]$. At the silicone oil-water interface, a pre-adsorption of PS20 is able to reduce protein adsorption significantly [15]. These differences reveal that, the protective efficacies of surfactants in preventing IgG1 surface adsorption strongly depend on complex material surface properties that cannot be described by a hydrophobic WCA measurement alone. Indeed, the hydrophobic materials considered in this study have WCAs ranging from $82^{\circ}$ (PC) to $103^{\circ}$ (C16), but there is no obvious correlation between the protective efficacy of the surfactant and the water contact angle of the materials (Supplementary Figure 5). The strength with which the surfactant (or protein) binds to a particular surface is likely to depend on a combination of properties like the nature and density of surface functional groups, the 
degree of crystallinity, the surface rugosity, and others. An indirect illustration of this is also given by the work of Browne and colleagues who demonstrated that on polystyrene, albumin adsorption decreased with an increasing oxidation state of the polymer surface [7]. In order to compare the potential of surfactant protection for mAbs on a variety of plastic materials, we developed an ELISA assay, that is versatile and rapid and can be implemented directly on the chosen plastic. It differs from the surfactant pre-coating protocols in that the surfactant and antibody are applied simultaneously to the surface, mimicking formulation conditions. Using this approach, we extended our adsorption studies to two other mAbs (mAbB and mAbC, Figure 3a) and to PS20 and P188 (Figure 3b), two other surfactants often used in therapeutic formulations. Varying PS80 concentrations $\left(0\right.$ to $\left.0.2 \mathrm{~g} . \mathrm{L}^{-1}\right)$ we show that, for each mAb at its respective formulation concentration, a significant difference in the amount of mAb surface adsorption is observed in a PS multi-well plate compared to octadecane (C18)-functionalized glass beads in a PEG-coated hydrophilic multi-well plate (Figure 3a). While the protection efficacy improves rapidly on PS with increasing PS80 concentrations for all antibodies tested (maximal protection at $0.2 \mathrm{~g} \cdot \mathrm{L}^{-1} \mathrm{PS} 80$ ), the protection efficacy of PS80 on C18 is minimal and does not improve significantly in the concentration range tested. This behaviour shows that on PS, contrary to C18, PS80 competes efficiently and dominates surface adsorption above a critical concentration. This has also been described for the protection efficacy of PS80 and lysozyme (0.5 g.L $\left.{ }^{-1}\right)$ adsorption on hydrophobic silica functionalized with dichlorodimethylsilane $\left(\right.$ WCA $\left.=90-110^{\circ}\right)$ for which maximal protection was measured at $0.08 \mathrm{~g} \cdot \mathrm{L}^{-1}$ (80ppm) [17]. When considering PS20 and more particularly $\mathrm{P} 188$ at $0.2 \mathrm{~g} \cdot \mathrm{L}^{-1}$, we show that their protection efficacies against $\mathrm{mAbA}$ surface adsorption is not significantly different than the one described for PS80 on both PS and C18 surfaces. In a study carried out on hydrophilic silica-titania surfaces, Kim and 
colleagues concluded that P188 inhibits protein adsorption by forming protein-surfactant complexes rather than by preferential surface adsorption which is the case for polysorbates [18]. It was also shown that PS20 was more effective than P188 to prevent mAb adsorption at the silicone oil-water interface [19]. Our results, based on the relative quantification of adsorbed mAbA on hydrophobic surfaces, do not suggest a different underlying protection mechanism for polysorbates and P188 and rather point towards equally efficient/inefficient surface interactions on PS/C18 for all surfactants tested.

We then quantitatively compared the protective effect of PS80 on different, commercially available plastic multi-well plates (Figure 4). The variability of the protection efficacy observed for the different plastic materials tested illustrates that a complex interplay of material properties dictates molecular surface binding for both proteins and surfactants and can thus result in strong or weak surfactant protection efficacies. Adsorption studies with "real materials" in use during the life cycle of a therapeutic molecule are quite rare because they are often not easily amenable to surface-sensing techniques. Zangmeister demonstrated by X-Ray photo-electron spectroscopy (XPS) that a mAb and a polyclonal human IgG could adsorb on a glass vial, a vial rubber cap liner, a syringe plunger tip, and a cell culture flask but not on PVC tubing nor on a plastic barrel of a syringe [20]. Different polymeric filter materials were tested for mAb and PS80 adsorption by Mahler and colleagues [21], who demonstrated that the amount of protein adsorption varies with filter material but also with different suppliers for the same material. In an attempt to control cell adhesion, the influence of surface functional groups in culture dish inserts made from various plastic polymers (PS, PC, polystryrene-co-acrylonitrile and polyetherimide) on IgG1 adsorption was analysed [22]. Despite similar wettability and surface roughness, surfaceadsorbed protein amounts varied, with PS showing minimal adsorption and PEI showing the 
highest protein adsorption. The authors conclude that the chemical composition of the polymers allows for specific combinations of potential interaction forces, influencing protein adsorption profiles. Zheng and colleagues drew similar conclusions by reviewing the influence of surface charge, chemical composition, surface topography and crystalline phases in hydrophilic bioactive glasses on BSA adsorption [23]. Interistingly, Thyparambil and colleagues also showed that surfactant performance in eluting adsorbed proteins from surfaces not only depends on the detergent used but also on the type of surface [24]. The consequences of differential surface adsorption in response to underlying material properties is also illustrated in different modes of surface-induced amyloid fibrillation [25].

\section{Conclusion}

Our study confirms that inherent material properties of plastic polymers influence protein but also surfactant adsorption in such a way that it can greatly affect the protective efficacy of commonly used surfactants such as polysorbates or poloxamer 188. It is therefore crucial to include the evaluation of protection efficacies at targeted material interfaces in the formulation optimisation process. If materials can be provided in pieces with fitting dimensions compatible with analysis in multiwell plates, our ELISA-based assay represents a convenient screening protocol in formulation optimization for different materials relevant to therapeutic protein handling and administration.

\section{Funding Statement}

GL was supported by an ANRT Cifre PhD grant (2017/0735). This work was supported by the French National Research Agency in the framework of the "Investissements d'avenir" program (ANR-15-IDEX-02). 


\section{Acknowledgements}

The authors thank Matthieu Weber for excellent technical expertise with XPS acquisition.

\section{Conflict of Interest Statement}

The authors declare no conflicts of interest.

\section{Author contributions}

GL developed the methodology and did the experimental work together with AM; VF and SH provided the molecules for the study; VF, SH, FB and $\mathrm{MW}$ are responsible for the supervision, the conceptualization, the formal analysis and the validation; GL and MW prepared the manuscript; VF and SH together with FB and MW secured the funding. 


\section{References}

1. Lu RM, Hwang YC, Liu IJ, Lee CC, Tsai HZ, Li HJ, et al. Development of therapeutic antibodies for the treatment of diseases. J Biomed Sci. 2020;27:1-30.

2. Kaplon H, Muralidharan M, Schneider Z, Reichert JM. Antibodies to watch in 2020. MAbs. Taylor and Francis Inc.; 2020;12:e1703531.

3. Moussa EM, Panchal JP, Moorthy BS, Blum JS, Joubert MK, Narhi LO, et al. Immunogenicity of Therapeutic Protein Aggregates. J Pharm Sci. 2016;105:417-30.

4. Khan TA, Mahler H-C, Kishore RSK. Key interactions of surfactants in therapeutic protein formulations: A review. Eur J Pharm Biopharm. 2015;97:60-7.

5. Gervasi V, Dall Agnol R, Cullen S, McCoy T, Vucen S, Crean A. Parenteral protein formulations: An overview of approved products within the European Union. Eur J Pharm Biopharm. 2018;131:8-24.

6. Zhang Z, Orski S, Woys AM, Yuan G, Zarraga IE, Wagner NJ, et al. Adsorption of polysorbate 20 and proteins on hydrophobic polystyrene surfaces studied by neutron reflectometry. Colloids Surfaces B Biointerfaces. 2018; 168:94-102.

7. Browne MM, Lubarsky G V., Davidson MR, Bradley RH. Protein adsorption onto polystyrene surfaces studied by XPS and AFM. Surf Sci. 2004;553:155-67.

8. Kapp SJ, Larsson I, Van De Weert M, Cárdenas M, Jorgensen L. Competitive adsorption of monoclonal antibodies and nonionic surfactants at solid hydrophobic surfaces. J Pharm Sci. 2015;104:593-601.

9. Couston RG, Skoda MW, Uddin S, Van Der Walle CF. Adsorption behavior of a human monoclonal antibody at hydrophilic and hydrophobic surfaces. MAbs. 2013;5:126-39. 10. Sacha GA, Saffell-Clemmer W, Abram K, Akers MJ. Practical fundamentals of glass, rubber, and plastic sterile packaging systems. Pharm Dev Technol. 2010;15:6-34. 
11. Le Basle Y, Chennell P, Tokhadze N, Astier A, Sautou V. Physicochemical Stability of Monoclonal Antibodies: A Review. J. Pharm. 2020; 109:169-90.

12. Lefebvre G, Bruckert F, Filipe V, Huille S, Weidenhaupt M. Adsorption rate constants of therapeutic proteins and surfactants for material surfaces. Colloids Surfaces B Biointerfaces 2021;203:111722.

13. Nault L, Guo P, Jain B, Brechet $Y$, Bruckert F, Weidenhaupt M. Human insulin adsorption kinetics, conformational changes and amyloidal aggregate formation on hydrophobic surfaces. Acta Biomater. 2013;9:5070-9.

14. Prime KL, Whitesides GM. Self-assembled organic monolayers: model systems for studying adsorption of proteins at surfaces. Science. 1991;252:1164-67.

15. Dixit N, Maloney KM, Kalonia DS. The effect of Tween ${ }^{\circledR} 20$ on silicone oil-fusion protein interactions. Int J Pharm. 2012;429:158-67.

16. Joshi O, McGuire J. Adsorption behavior of lysozyme and tween 80 at hydrophilic and hydrophobic silica-water interfaces. Appl Biochem Biotechnol. 2009;152:235-48.

17. Joshi O, McGuire J. Adsorption behavior of lysozyme and Tween 80 at hydrophilic and hydrophobic silica-water interfaces. Appl Biochem Biotechnol. 2009;152:235-48.

18. Kim HL, Mcauley A, Livesay B, Gray WD, Mcguire J. Modulation of Protein Adsorption by Poloxamer 188 in Relation to Polysorbates 80 and 20 at Solid Surfaces. J Pharm Sci . 2014;103:1043-9.

19. Kannan A, Shieh IC, Negulescu PG, Chandran Suja V, Fuller GG. Adsorption and Aggregation of Monoclonal Antibodies at Silicone Oil-Water Interfaces. Mol Pharm. 2021;18:1656-65.

20. Zangmeister RA. Application of x-ray photoelectron spectroscopic analysis to protein adsorption on materials relevant to biomanufacturing. J Pharm Sci. 2012;101:1639-44. 
21. Mahler H-C, Huber F, Kishore RSK, Reindl J, Rückert P, Müller R. Adsorption Behavior of a Surfactant and a Monoclonal Antibody to Sterilizing-Grade Filters. J Pharm Sci. 2010;99:2620-7.

22. Vijaya Bhaskar TB, Roch T, Romero O, Ma N, Kratz K, Lendlein A. Single and competitive protein adsorption on polymeric surfaces. Polym Adv Technol. 2015;26:1387-93.

23. Zheng K, Kapp M, Boccaccini AR. Protein interactions with bioactive glass surfaces: $A$ review. Appl Mater Today. 2019;15:350-71.

24. Thyparambil AA, Wei Y, Latour RA. Evaluation of the Effectiveness of Surfactants and Denaturants to Elute and Denature Adsorbed Protein on Different Surface Chemistries. Langmuir. 2015;31:11814-24.

25. Grigolato F, Arosio P. The role of surfaces on amyloid formation. Biophys Chem. 2021;270:106533. 


\section{Figures}
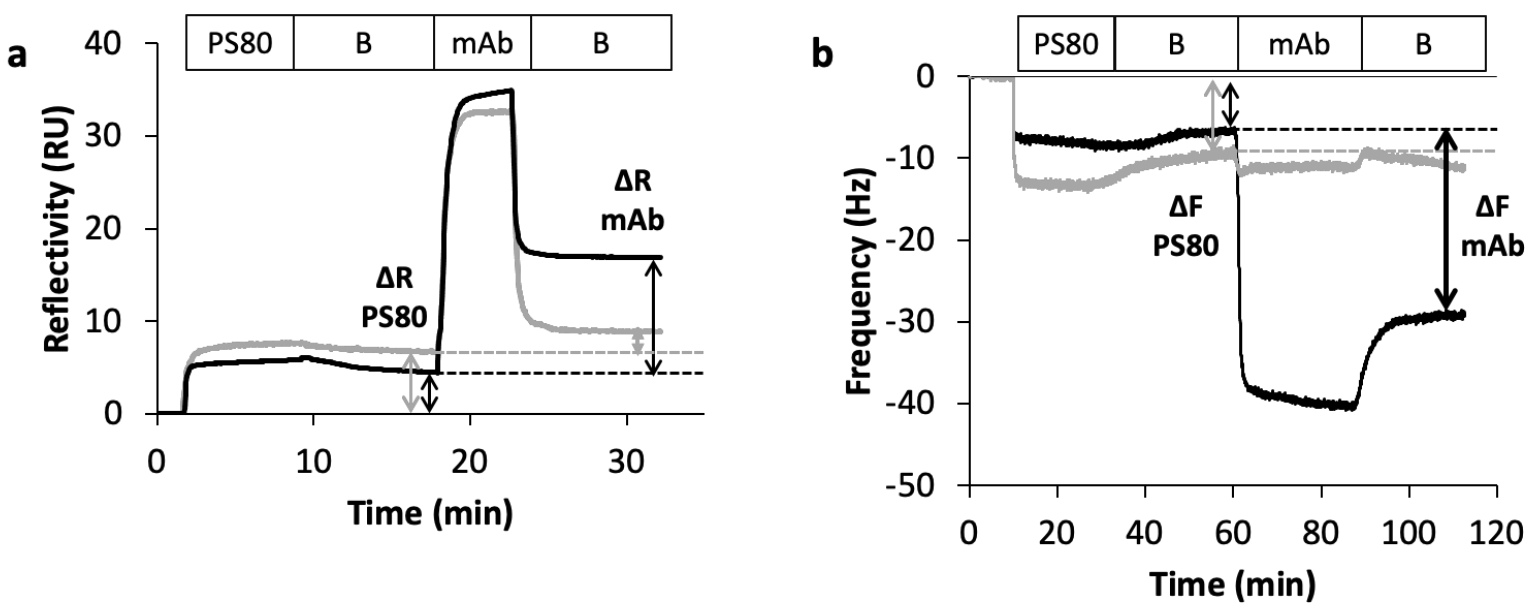

Fig 1 Adsorption of PS80 and mAbA on polystyrene (PS) and on a hexadecane (C16) monolayer

The top panel shows the solution present in the flow chamber at the different time points (PS80, $B=$ buffer, $m A b=m A b A)$.

a, Adsorption recorded by SPRi during a sequential injection of PS80 at 0.2 g.L-1 and mAbA at 30 g.L-1 on a prism functionalized with polystyrene (grey) or hexadecane (black). $\Delta R$ PS80 and $\triangle \mathrm{R}$ mAbA (double headed arrows) indicate the amount of PS80 and mAbA that remain adsorbed on the surfaces after a buffer wash, expressed in reflectivity units, RU (see Table 1).

b, Adsorption recorded by QCM-d during a sequential injection of PS 80 at 0.2 g.L-1 and mAbA at 30 g.L-1 on a sensor functionalized with polystyrene (grey) or hexadecane (black). $\triangle \mathrm{F} \mathrm{PS} 80$ and $\triangle \mathrm{F} \mathrm{mAbA}$ (double headed arrows) indicate the amount of PS80 and $\mathrm{mAbA}$ that remain adsorbed on the surfaces after a buffer wash, expressed in Hertz, $\mathrm{Hz}$ (see Table I). 

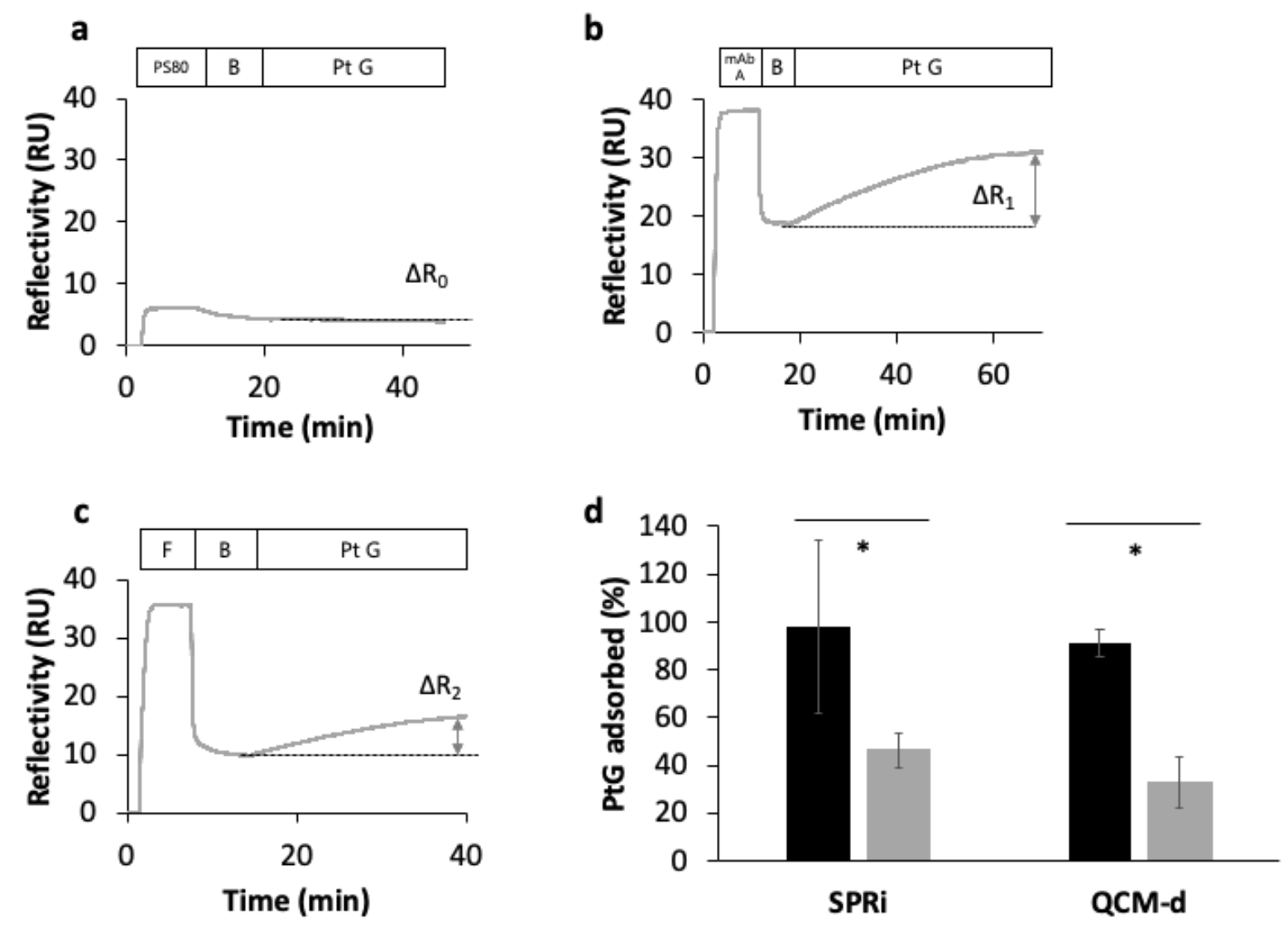

Fig 2 Effect of PS80 on the adsorption of mAbA to polystyrene or a hexadecanefunctionalized surface

SPRi adsorption kinetics showing the PtG-associated reflectivity signal obtained on a polystyrene-coated prism. The PtG is injected after: $a$, an adsorption of PS80 at $0.2 \mathrm{~g} \cdot \mathrm{L}^{-1}\left(\Delta \mathrm{R}_{0}\right)$ followed by a buffer (B) wash, b, an adsorption of mAbA at $30 \mathrm{~g} \cdot \mathrm{L}^{-1}$ without surfactant $\left(\Delta \mathrm{R}_{1}\right)$ followed by a buffer (B) wash and c, an adsorption of $\mathrm{mAbA}$ at $30 \mathrm{~g} \cdot \mathrm{L}^{-1}$ with PS80 at $0.2 \mathrm{~g} \cdot \mathrm{L}^{-1}$ (formulation condition $(F))\left(\Delta R_{2}\right)$ followed by a buffer $(B)$ wash. $d$, Plot showing the amount of adsorbed PtG in formulation conditions normalized to the condition without surfactant on C16 (black) and PS (grey) by SPRi (left) and QCM-d (right). The results are expressed according to the formula $\left(\Delta R_{2}-\Delta R_{0}\right) / \Delta R_{1}$ for the SPRi data and likewhise to the formula $\left(\Delta F_{2^{-}}\right.$ $\left.\Delta \mathrm{F}_{0}\right) / \Delta \mathrm{F}_{1}$ for the QCM-d data (Supplementary Figure 1 and Supplementary Table I). Each bar corresponds to the mean of $\mathrm{n}$ experiments and the error bars indicate the standard 
deviation. ${ }^{*} p=0.11$ (ANOVA, $n=3$ ) for SPRi results and $p=0.012$ (ANOVA, $n=2$ ) for QCM-d results. 

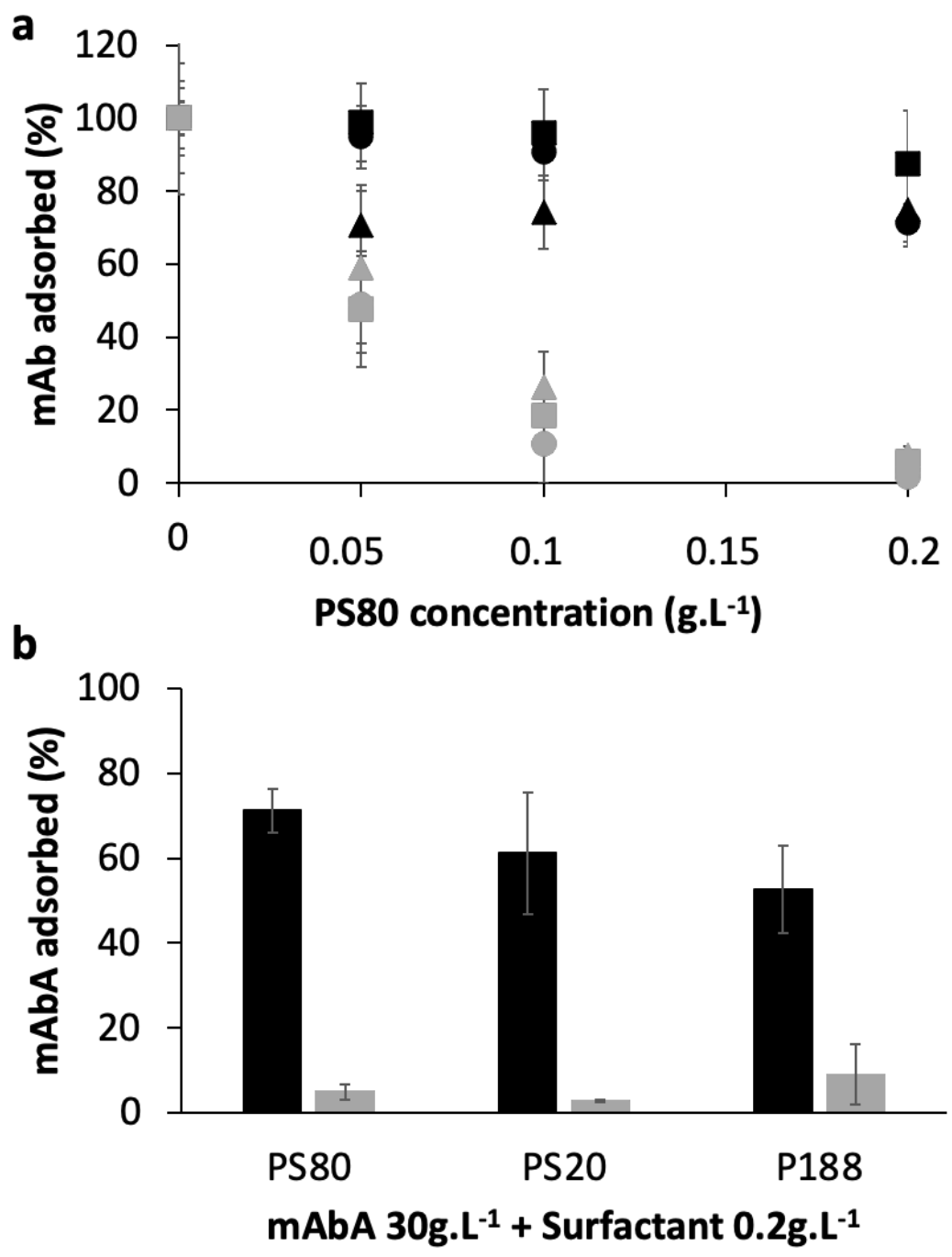

Fig 3 Comparison of the protective effect of different surfactants and mAbs on polystyrene and on a polycarbon surface functionalization

a, Normalized adsorption of $\mathrm{mAbA}$ at $30 \mathrm{~g} . \mathrm{L}^{-1}$ (circles), $\mathrm{mAbB}$ at $0.7 \mathrm{~g} \cdot \mathrm{L}^{-1}$ (triangles) and $\mathrm{mAbC}$ at 3 g. $\mathrm{L}^{-1}$ (squares) on octadecane (C18)-functionalized glass beads (black) and on polystyrene plates (grey) as a function of PS80 concentration in solution.

b, Normalized adsorption of mAbA (30 g.L-1) on C18 (black) and polystyrene (grey) surfaces in the presence of 0.2 g.L-1 PS80, PS20 and P188 in solution.

mAb adsorption is quantified by ELISA using a PtG-HRP construct. Data are obtained by absorbance measurements using a colorimetric enzymatic readout. They are normalized to the absorbance measured for $\mathrm{mAbA}$ adsorption without surfactant. Each data point 
corresponds to the mean of eight experiments and the error bars indicate the standard deviation. 


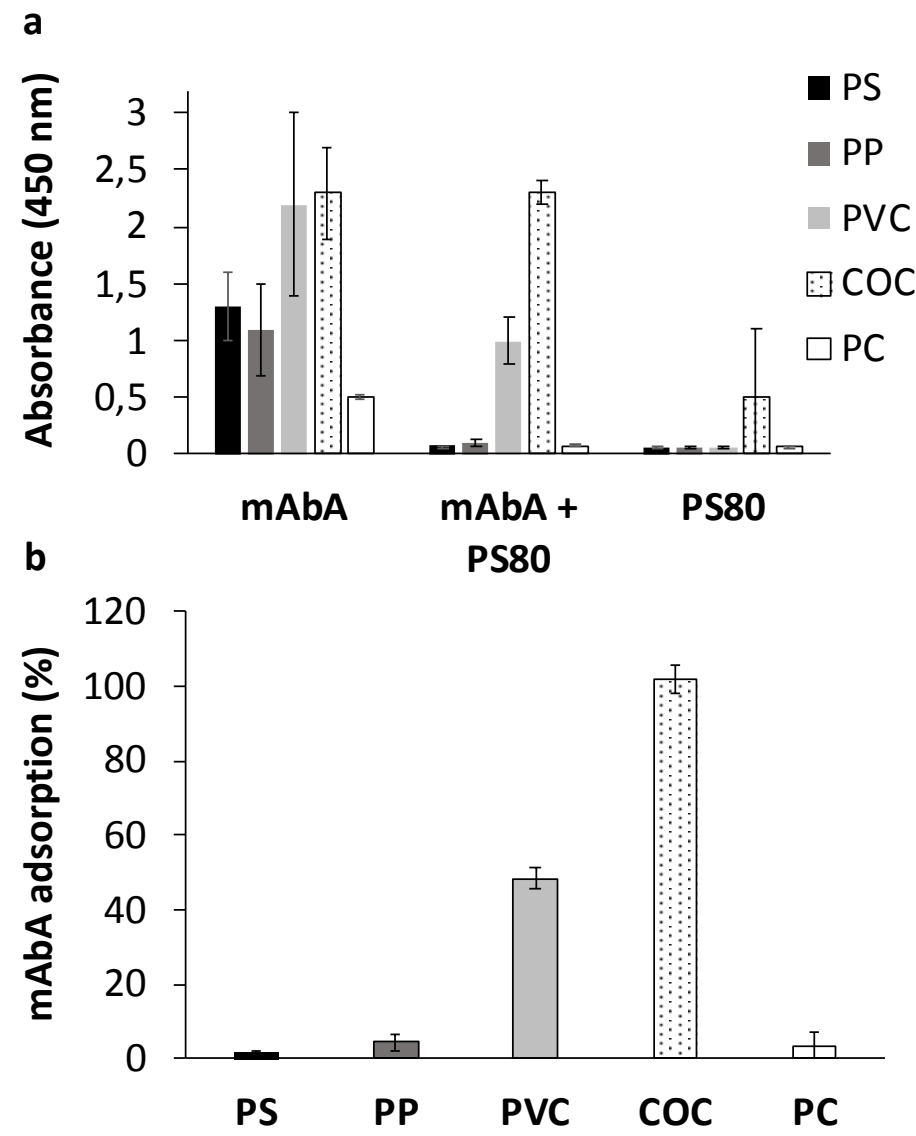

Fig 4 PS80 protective effect on different material surfaces

mAbA adsorption is quantified by ELISA using a PtG/A-HRP construct. Data are obtained by absorbance measurements using a colorimetric readout at $450 \mathrm{~nm}$.

a, Absorbance at $450 \mathrm{~nm}$ for mAbA $\left(30 \mathrm{~g} . \mathrm{L}^{-1}\right)$ adsorption without and with PS80 (0.2 g.L $\left.\mathrm{L}^{-1}\right)$ and for PS80 (0.2 g. $\left.\mathrm{L}^{-1}\right)$ alone, in a polystyrene plate (PS, black), a polypropylene plate (PP, dark grey), a polyvinyl chloride plate (PVC, light grey), a cyclic olefin copolymer plate (COC, dotted) and a polycarbonate plate (PC, white). For the COC plate, a PtA-HRP construct was used.

b, Bar diagram illustrating the protective effect of PS80 on the different plastic surfaces. Each bar represents the \% of $\mathrm{mAbA}$ adsorbed in the presence of $0.2 \mathrm{~g} \cdot \mathrm{L}^{-1} \mathrm{PS} 80$, normalized to the condition without surfactant. The absorbance data of panel a were used for this 
representation. Each data point corresponds to the mean of eight experiments and the error bars indicate the standard deviation. 


\section{Table}

Table I Reflectivity and frequency data for adsorbed mAb and surfactant on different surfaces

\begin{tabular}{|c|c|c|}
\hline SPR Surface $^{\mathrm{a}}$ & $\Delta \mathbf{R}_{\mathrm{PS80}}(\mathbf{R U})^{\mathrm{b}}$ & $\Delta \mathbf{R}_{\mathrm{mAbA}}-\Delta \mathbf{R}_{\mathrm{PS} 80}(\mathrm{RU})^{\mathbf{b}}$ \\
\hline Polystyrene & $6.5 \pm 2.5$ & $2 \pm 2$ \\
\hline C16 & $5 \pm 1.5$ & $11.5 \pm 1.5$ \\
\hline QCM-d Surface $^{\mathrm{a}}$ & $\Delta \mathrm{F}_{\mathrm{PS80}}(\mathrm{Hz})^{\mathrm{c}}$ & $\Delta \mathrm{F}_{\mathrm{mAbA}}-\Delta \mathrm{F}_{\mathrm{PS} 80}(\mathrm{~Hz})^{\mathbf{c}}$ \\
\hline Polystyrene & $-11.5 \pm 1.5$ & $-3.5 \pm 3$ \\
\hline C16 & $-7.5 \pm 0.5$ & $-18 \pm 0.5$ \\
\hline
\end{tabular}

${ }^{a}$ Adsorption of PS80 and mAbA on sensor surfaces functionalized with polystyrene and hexadecane as measured by SPRi and QCM-d (see Figure 1).

${ }^{\mathrm{b}}$ Each SPRi value corresponds to the mean of three experiments and their standard deviation. ${ }^{\mathrm{C}}$ Each QCM-d value corresponds to the mean of two experiments and their standard deviation. 


\section{Supplementary Materials}

\section{Supplementary Figure 1}
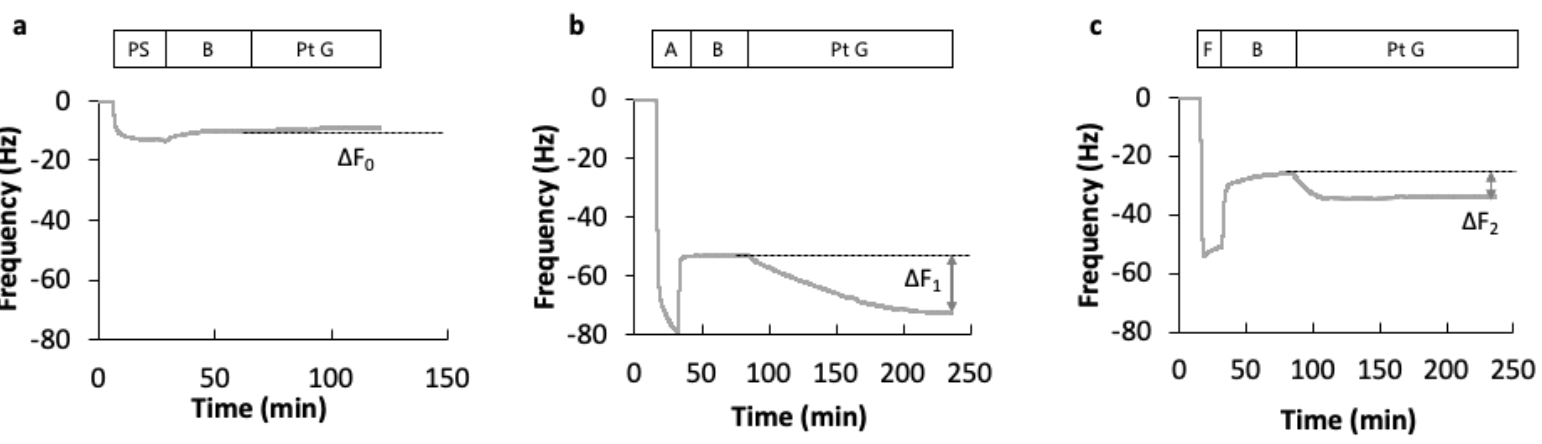

Supplementary Fig 1 mAbA adsorption on polystyrene measured by PtG frequency shifts using QCM-d

QCM-d adsorption kinetics showing the PtG-associated frequency signal obtained on a polystyrene-functionalized sensor. The PtG is injected after: a, an adsorption of PS80 at 0.2g. $\mathrm{L}^{-1}\left(\Delta \mathrm{F}_{0}\right)$ followed by a buffer $(\mathrm{B})$ wash, $\mathrm{b}$, an adsorption of $\mathrm{mAbA}(\mathrm{A})$ at $30 \mathrm{~g} \cdot \mathrm{L}^{-1}$ without surfactant $\left(\Delta \mathrm{F}_{1}\right)$ followed by a buffer $(\mathrm{B})$ wash and $\mathrm{c}$, an adsorption of $\mathrm{mAbA}$ at $30 \mathrm{~g} \cdot \mathrm{L}^{-1}$ with PS80 at $0.2 \mathrm{~g} \cdot \mathrm{L}^{-1}$ (formulation condition, $\left.\left.\mathrm{F}\right)\right)\left(\Delta \mathrm{F}_{2}\right)$ followed by a buffer $(\mathrm{B})$ wash. 
Supplementary Table I PtG reflectivity and frequency values measured on hexadecane and polystyrene surfaces

\begin{tabular}{|c|c|c|c|}
\hline SPR Surface $^{\mathrm{a}}$ & $\Delta \mathbf{R}_{\mathbf{0}}(\mathrm{RU})^{\mathrm{b}}$ & $\Delta \mathrm{R}_{\mathbf{1}}(\mathrm{RU})^{\mathrm{b}}$ & $\Delta \mathbf{R}_{\mathbf{2}}(\mathrm{RU})^{\mathrm{b}}$ \\
\hline Polystyrene & $0.3 \pm 0.3$ & $12.3 \pm 2.2$ & $5.7 \pm 0.8$ \\
\hline C16 & $0.3 \pm 0.3$ & $6.6 \pm 0.1$ & $6.4 \pm 2.4$ \\
\hline QCM-d Surface $^{\mathrm{a}}$ & $\Delta \mathrm{F}_{\mathbf{0}}(\mathrm{Hz})^{\mathrm{c}}$ & $\Delta \mathrm{F}_{\mathbf{1}}(\mathrm{Hz})^{\mathrm{c}}$ & $\Delta \mathrm{F}_{\mathbf{2}}(\mathrm{Hz})^{\mathrm{c}}$ \\
\hline Polystyrene & $0.5 \pm 0.5$ & $20 \pm 1.5$ & $6.5 \pm 2.1$ \\
\hline C16 & $0.5 \pm 0.5$ & $19.5 \pm 2.1$ & $17.7 \pm 1.1$ \\
\hline
\end{tabular}

${ }^{\text {a }}$ Adsorbed PtG amounts on sensor surfaces functionalized with hexadecane (C16) and polystyrene measured by SPRi and QCM-d (see Figure 1 and Supplementary Figure 1).

b Each SPRi value corresponds to the mean of three experiments and their standard deviation. ${ }^{c}$ Each QCM-d value corresponds to the mean of two experiments and their standard deviation. 

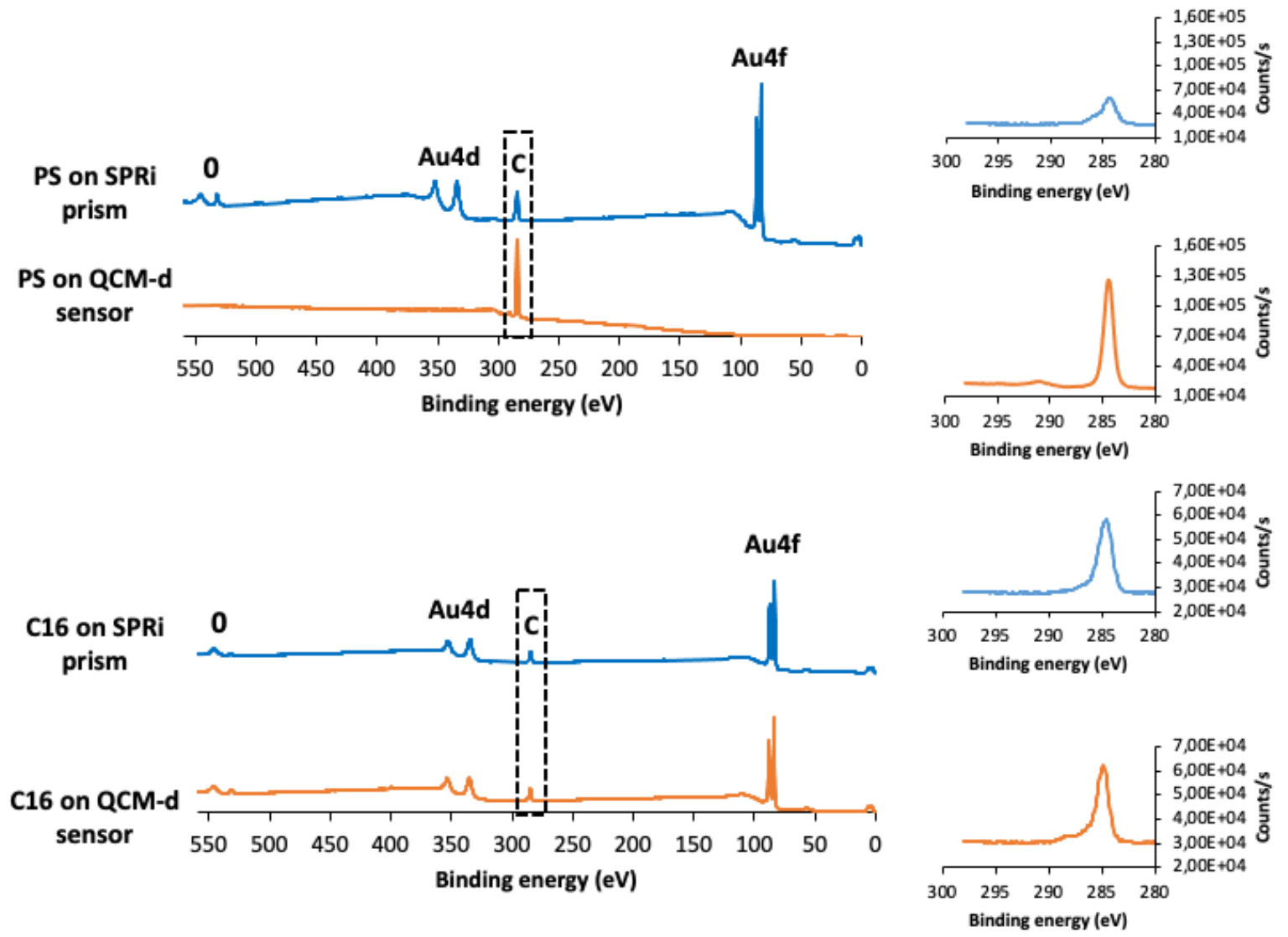

\section{Supplementary Fig 2 Characterisation of polystyrene and C16 surface functionalizations}

Top, X-ray photoelectron spectroscopy (XPS) analysis of an SPRi prism (blue), functionalized with polystyrene-thiol (PS-SH) and a purchased QCM-d sensor, spin-coated with PS (QSX305, Biolin Scientific). The characteristic C peak around $285 \mathrm{eV}$ (dotted box) is shown enlarged on the right of the main spectra.

Bottom, XPS analysis of an SPRi prism (blue) and a QCM-d sensor (orange), functionalized both with hexadecane-thiol (C16-SH). The characteristic C peak around $285 \mathrm{eV}$ (dotted box) is shown enlarged on the right of the main spectra. 


\section{Supplementary Figure 3}

a

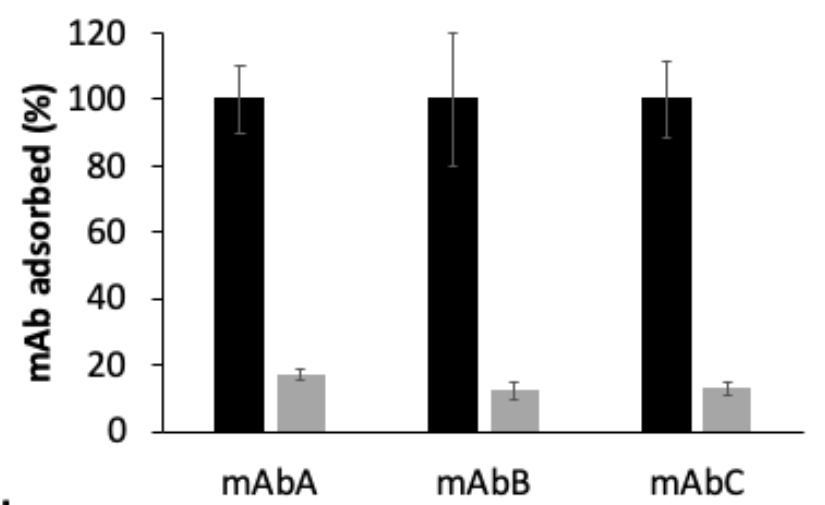

b

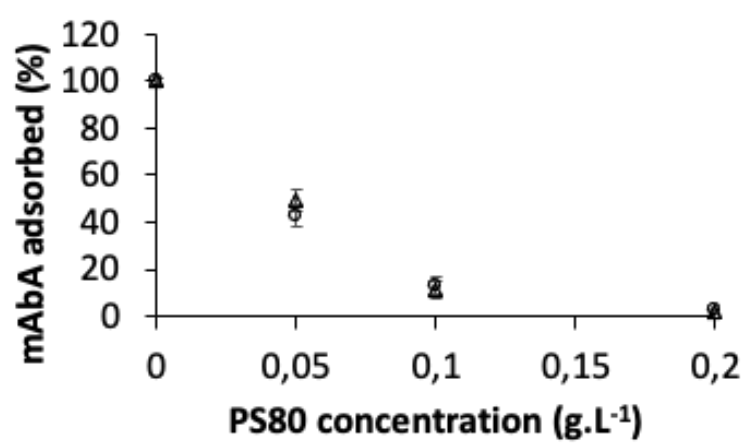

Supplementary Fig 3 mAb adsorption on octadecane-functionalized glass beads in PEGcoated plates and on polystyrene plates using PtG and PtA-HRP constructs

a, Comparison of mAbA at $30 \mathrm{~g} . \mathrm{L}^{-1}, \mathrm{mAbB}$ at $0.7 \mathrm{~g} \cdot \mathrm{L}^{-1}$ and $\mathrm{mAbC}$ at $3 \mathrm{~g} \cdot \mathrm{L}^{-1}$ adsorbed on PEGcoated wells (grey) and on PEG-coated wells containing octadecane-functionalized glass beads (black). mAb adsorption is quantified by ELISA using a PtG-HRP construct. Data are obtained by absorbance measurements using a colorimetric enzymatic readout. They are normalized to the absorbance measured for $\mathrm{mAbA}, \mathrm{mAbB}$ and $\mathrm{mAbC}$ adsorption on octadecane-functionalized glass beads. Each bar corresponds to the mean of eight experiments and the error bars indicate the standard deviation.

b, mAbA (30 g. $\left.\mathrm{L}^{-1}\right)$ adsorbed on a polystyrene plate as a function of PS80 concentration quantified by ELISA using a PtG-HRP construct (circles) or a PtA-HRP construct (triangles). Data are obtained by absorbance measurements using a colorimetric enzymatic readout and 
normalized to the absorbance measured for mAbA adsorption without surfactant. Each data point corresponds to the mean of eight experiments and the error bars indicate the standard deviation. 


\section{Supplementary Figure 4}

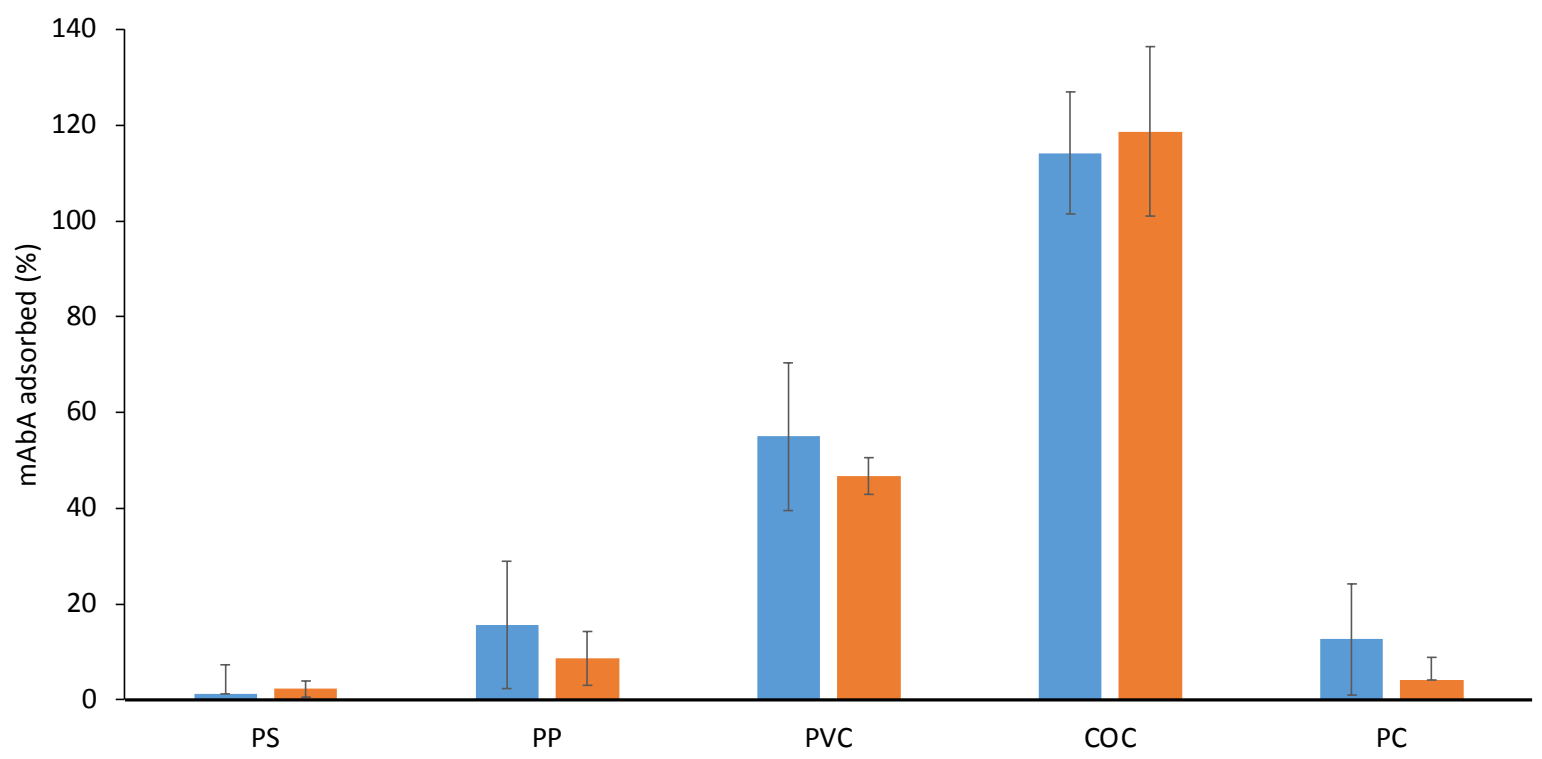

Supplementary Fig 4: Comparison of the protective effect of PS80 from Seppic and Croda on different plastic surfaces

Bar diagram illustrating the protective effect of PS80 from Seppic (blue) or Croda (orange) on the different plastic surfaces. Each bar represents the \% of mAbA (30g. $\mathrm{L}^{-1}$ ) adsorbed in the presence of $0.2 \mathrm{~g} \cdot \mathrm{L}^{-1} \mathrm{PS} 80$, normalized to the condition without surfactant. The experimental protocol is identical to the one described in the manuscript. Polystyrene (PS), polypropylene (PP), polyvinyl chloride (PVC), cyclic olefin copolymer (COC) and polycarbonate (PC) surfaces correspond to the same multiwell plates as those described in the manuscript. Each data point corresponds to the mean of eight replicas and the error bars indicate the standard deviation. 


\section{Supplementary Figure 5}

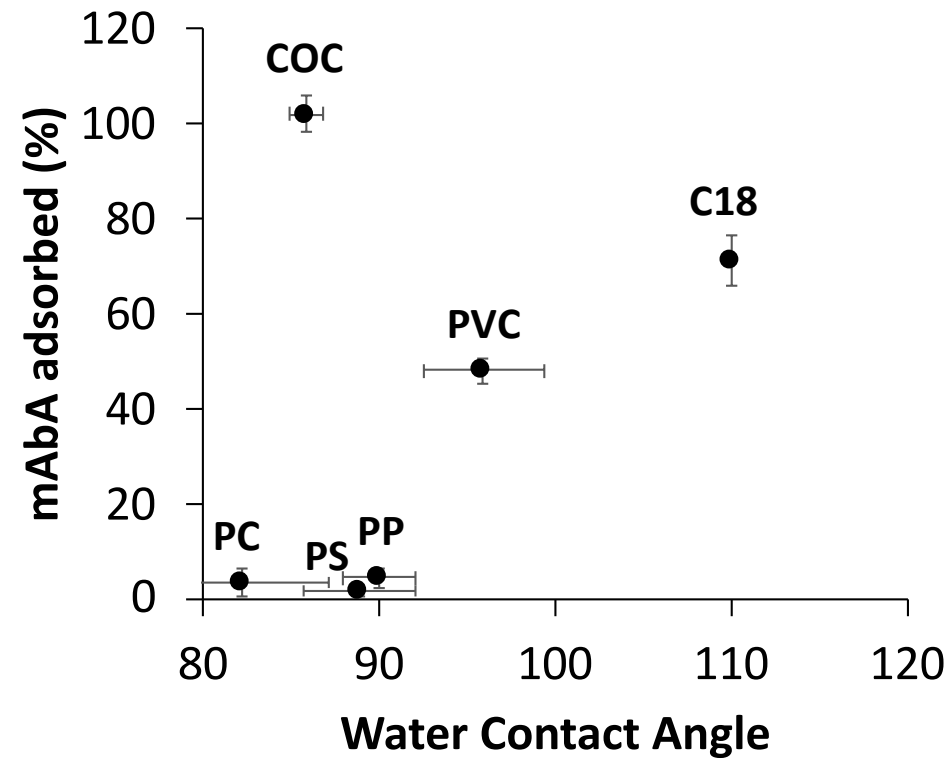

Supplementary Fig 5 Protection efficacy of PS80 on mAbA surface adsorption as a function of water contact angle of the materials

Plot showing the amount of mAbA $\left(30 \mathrm{~g} \cdot \mathrm{L}^{-1}\right)$ adsorbed in the presence of PS80 at $0.2 \mathrm{~g} \cdot \mathrm{L}^{-1}$ on different materials as a function of the WCAs of the materials. The data were obtained by the ELISA assay and are normalized to the amount of mAbA adsorption in the absence of surfactant. The adsorption datapoints represent mean values calculated from 8 replicates in 2 independent experiments and their standard deviation. The WCA datapoints represent mean values from 4 replicate measurements (except for $\mathrm{C} 18$, one replicate) and their standard deviation. 